\title{
An Independent and Coordinated Criterion for Kinematic Aircraft Maneuvers
}

\author{
Anthony Narkawicz César A. Muñoz* \\ George Hagen \\ NASA Langley Research Center, Hampton, VA, 23881, US
}

\begin{abstract}
This paper proposes a mathematical definition of an aircraft-separation criterion for kinematic-based horizontal maneuvers. It has been formally proved that kinematic maneuvers that satisfy the new criterion are independent and coordinated for repulsiveness, i.e., the distance at closest point of approach increases whether one or both aircraft maneuver according to the criterion. The proposed criterion is currently used in NASA's Airborne Coordinated Resolution and Detection (ACCoRD) set of tools for the design and analysis of separation assurance systems.
\end{abstract}

\section{Nomenclature}

D Minimum horizontal separation

$\epsilon \quad$ Directionality parameter whose value is \pm 1

s Two-dimensional aircraft position

$t \quad$ Time variable

$\tau \quad$ Time step

$\boldsymbol{u}, \mathbf{w} \quad$ Two-dimensional vector variables

$\mathbf{v} \quad$ Two-dimensional aircraft velocity

V Kinematic maneuver

Subscripts

$o \quad$ Ownship information of a position or velocity vector

$i \quad$ Intruder information of a position or velocity vector

$x \quad$ Northern component of a position or velocity vector

$y \quad$ Eastern component of a position or velocity vector

\section{Introduction}

Maintaining aircraft separation is a safety objective of air traffic management systems. A credible demonstration that this goal has been achieved for a given airspace concept is challenging due to many inherent characteristics of these concepts. In particular, advanced airspace concepts often involve safety-critical distributed software that interacts with human actors [9]. The standard method of demonstrating the viability of an advanced airspace concept is through high fidelity simulations of relevant scenarios. However, no matter how many scenarios are examined, this approach can never demonstrate that the safety-critical software, on which the concept is based, keeps aircraft separated in all scenarios, including those that are off-nominal. Given the paramount importance of safety, an approach that can give strong assurances for the safe behavior of new air traffic management systems is necessary when developing such systems.

In previous work by the authors [4], an analytical and formal approach for verifying the safe interplay between (possibly different) separation assurance algorithms was proposed. This approach relies on the definitions of analytical formulas, called criteria, that characterize sets of resolution maneuvers that are both independent, i.e., separation is ensured when only one aircraft maneuvers, and coordinated, i.e., separation

${ }^{*}$ Corresponding Author. Email: cesar.a.munoz@nasa.gov. AIAA Member. 
is ensured when two conflicting aircraft both maneuver. A criterion in this context is a formal condition that a proposed maneuver for an aircraft can satisfy. In a conflict scenario between two aircraft, if the resolution maneuvers of the aircraft each satisfy an appropriate criterion, it can be guaranteed that the aircraft resolve the conflict even if they simultaneously maneuver. It has been formally proved that for state-based (instantaneous) maneuvers, the given criteria provide sufficient conditions for independence and coordination [5]. Hence, resolution algorithms that compute state-based maneuvers that satisfy the criteria inherit independence and coordination properties regardless of how these maneuvers are computed.

The criteria presented by the authors in previous work can be used to show safe interplay between algorithms that give instantaneous maneuvers to the pilot. These state-based maneuvers are often computed by tactical conflict-resolution algorithms [3] for time to conflict that ranges between 5 and 20 minutes. For these times, trajectory prediction errors introduced by the instantaneous-maneuver assumption of statebased algorithms are considered acceptable. However, for short time intervals, such as in the case of collision avoidance systems [7] or loss of separation recovery algorithms [1], the instantaneous-maneuver assumption may produce infeasible or incorrect resolutions.

This paper extends the work in [5] by studying a criterion that does not assume instantaneous maneuvers. Indeed, the proposed criterion applies to kinematic-based horizontal maneuvers. The result is a mathematical criterion for kinematic maneuvers that guarantees independence and coordination. This coordination is implicit in the sense that no communication is assumed between the aircraft other than knowledge of the other aircraft's initial position and velocity before the maneuver. The proposed criterion is currently used in NASA's Airborne Coordinated Resolution and Detection (ACCoRD) ${ }^{\mathrm{a}}$ set of tools for the design and safety analysis of separation assurance systems.

The theorems in this paper have been formally proved in the Prototype Verification System (PVS) [6]. PVS is a system for verifying mathematical statements. In particular, PVS allows safety properties, such as independence and coordination, to be stated and formally proved. Once a safety property is specified in PVS, it is up to the user to prove in PVS that the condition holds. PVS has a reasoning engine that encodes mathematical logic, so only statements that are mathematically correct can be proved in PVS. The use of a system like PVS for proving safety properties about separation assurance systems in the airspace is justified by the importance of safety and the key part that these systems play in the overall safety of the airspace.

The rest of this paper is structured as follows. Section II gives a brief overview of the criteria-based resolution approach, Section III reviews coordination for instantaneous maneuvers, Section IV presents a criterion for kinematic maneuvers and its main properties, Section V provides a counterexample that shows that even trajectories that are incrementally diverging from one another do not always exhibit coordination, and Section VI concludes the paper.

\section{Criteria-Based Resolution Approach}

Formally, a criterion is specified as an analytical formula that defines a set of acceptable resolution maneuvers for an aircraft in a conflict scenario. As a simple example, a criterion specifically for aircraft in a head-on conflict might state that each aircraft must alter course to the right, as stated in part 91.113 of the Federal Aviation Regulations [2].

In general, criteria for instantaneous maneuvers can be analytically expressed by formulas involving the positions and velocity vectors of two aircraft, referred to as the ownship and the intruder, and a parameter that indicates directionality of the set of maneuvers, e.g., left/right, up/down, etc. For instance, a criterion for horizontal conflict avoidance was presented in [5]. That criterion specifies a mathematical condition on resolution maneuvers that are guaranteed to be independent, i.e., the maneuvers avoid the conflict assuming that only the ownship maneuvers, and coordinated, i.e., they also avoid the conflict if both aircraft simultaneously maneuver according to the criterion. The parameter indicating directionality for this criterion is called $\epsilon$, which takes a value of either -1 or 1 . If both aircraft maneuver, then they must select resolutions using the same $\epsilon$ value, which can be chosen implicitly from the input states of the aircraft. This is illustrated in Figure 1. The current ownship velocity vector is shown in blue and the current traffic velocity vector is shown in magenta. Conflict Detection and Resolution (CD\&R) systems that for both aircraft select resolution vectors anywhere in their green regions will produce resolution maneuvers that are always implicitly coordinated. Conversely, if the CD\&R systems on both aircraft select resolution vectors anywhere in their blue regions, the combined result will be implicitly coordinated. If only one aircraft maneuvers, then a vector

\footnotetext{
${ }^{a}$ http://shemesh.larc.nasa.gov/fm/ACCoRD/
} 


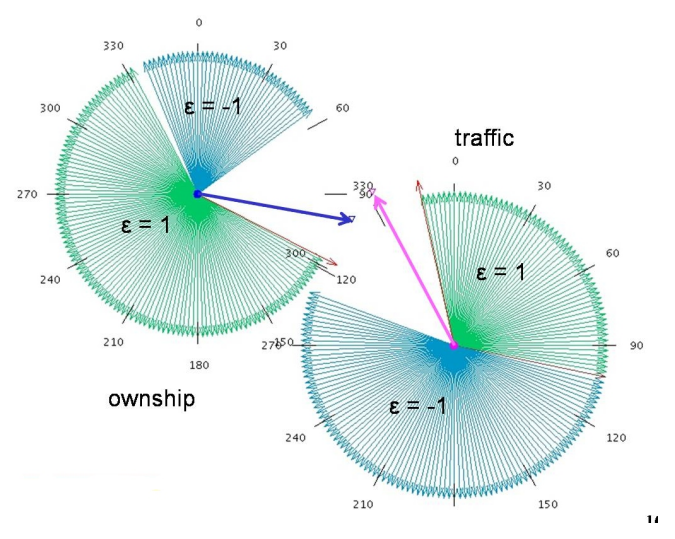

Figure 1. Visualization of Horizontal Criterion for Conflict Avoidance

in either the blue or green region will suffice.

The criterion for kinematic maneuvers proposed in this paper builds upon another criterion for instantaneous maneuvers that ensures repulsiveness. A resolution maneuver is repulsive if it increases the distance at the closest point of approach between two conflicting aircraft. The notion of repulsiveness is particularly relevant when a conflict between two aircraft can not be immediately solved. In this case, it is important that any resolution maneuver is in the appropriate direction, so that the distance at the closest point of approach increases even when both aircraft simultaneously maneuver. Similarly, the directions of the maneuvers are most important in a close encounter scenario such as when two aircraft have already lost minimum separation and must maneuver to recover separation. To eventually solve the conflict in these scenarios, aircraft have to maneuver in a way that incrementally improves the situation, which is measured by the distance at closest point of approach, hence the relevance of repulsiveness.

In [5], a criterion for instantaneous maneuvers that guarantees repulsiveness is proposed. The next section presents the basic definitions of the criteria-based approach for instantaneous maneuvers provided in that work.

\section{Coordination for Instantaneous Maneuvers}

In this paper, vector variables are written in boldface and can be denoted by their components. For example, if $\boldsymbol{u}$ is a 2-dimensional (2-D) vector, then $\boldsymbol{u}$ denotes the pair $\left(u_{x}, u_{y}\right)$. The two-dimensional Euclidean norm of the vector $\boldsymbol{u}$ is denoted by

$$
\|\boldsymbol{u}\| \equiv \sqrt{u_{x}^{2}+u_{y}^{2}}
$$

and the dot product of the 2-D vectors $\boldsymbol{u}$ and $\mathbf{w}$ is denoted

$$
\boldsymbol{u} \cdot \mathbf{w} \equiv\left(u_{x} w_{x}+u_{y} w_{y}\right)
$$

Furthermore, $\mathbf{0}$ denotes the zero vector, i.e., $\mathbf{0} \equiv(0,0)$ and $\boldsymbol{u}^{\perp}$ denotes the right perpendicular vector to $\boldsymbol{u}$, i.e.,

$$
\boldsymbol{u}^{\perp} \equiv\left(u_{y},-u_{x}\right)
$$

From these definitions, it can be easily proved that $\boldsymbol{u} \cdot \boldsymbol{u}^{\perp}=0$. The function det is defined by

$$
\operatorname{det}(\boldsymbol{u}, \mathbf{w}) \equiv \boldsymbol{u} \cdot \mathbf{w}^{\perp} .
$$

This paper considers pairwise resolution algorithms that return guidance maneuvers for aircraft. The terms ownship and intruder are used to distinguish between the aircraft for which the resolution maneuver is computed. These designations are relative in the sense that each aircraft will be from its point of view the ownship and the other aircraft will be the intruder. Without loss of generality, the development presented here takes the point of view of one of the aircraft, and that aircraft will be designated as the ownship. The algorithms discussed here use state-based information for the two aircraft as inputs, i.e., position and velocity 
vectors that are elements of a Euclidean space. The current states of the ownship and intruder aircraft at time $t=0$ are denoted by the following vectors:

- $\mathbf{s}_{o}, \mathbf{v}_{o}$ : Initial position and velocity of the ownship aircraft, respectively.

- $\mathbf{s}_{i}, \mathbf{v}_{i}$ : Initial position and velocity of the intruder aircraft, respectively.

Furthermore, it is assumed that the current ground speeds of the ownship and intruder aircraft are not zero, i.e., $\left\|\mathbf{v}_{o}\right\| \neq 0$ and $\left\|\mathbf{v}_{i}\right\| \neq 0$. Therefore, $\mathbf{v}_{o} \neq \mathbf{0}$ and $\mathbf{v}_{i} \neq \mathbf{0}$.

In the airspace system, the separation requirement for two aircraft is specified by a minimum horizontal separation $D$ (typically, $D$ is 5 nautical miles). A loss of separation between two aircraft occurs when the distance between them is less than $D$. Thus, the ownship and intruder aircraft are in loss of separation if and only if it holds that

$$
\left\|\mathbf{s}_{o}-\mathbf{s}_{i}\right\|<D \text {. }
$$

The separation requirement can be understood as an imaginary circle of diameter $D$ around each aircraft, and a conflict between two aircraft as a future overlapping of these circles. In this paper, an alternative but equivalent view is considered where the intruder is surrounded by a circle, called the protected zone, of radius $D$. From this perspective, a conflict between the ownship and intruder aircraft is defined as the existence of a time $t \geq 0$ at which the ownship is in the interior of the intruder's protected zone. In conflict detection algorithms, it is also required that $t$ is within a specified lookahead time. However, since this work concerns resolution algorithms, a lookahead time is not considered. Formally, the ownship and intruder aircraft are in conflict along their current linear trajectories if and only if there exists $t \geq 0$ such that, at time $t$, separation is lost, i.e., $\left\|\left(\mathbf{s}_{o}+t \mathbf{v}_{o}\right)-\left(\mathbf{s}_{i}+t \mathbf{v}_{i}\right)\right\|<D$. Since $\left(\mathbf{s}_{o}+t \mathbf{v}_{o}\right)-\left(\mathbf{s}_{i}+t \mathbf{v}_{i}\right)=\left(\mathbf{s}_{o}-\mathbf{s}_{i}\right)+t\left(\mathbf{v}_{o}-\mathbf{v}_{i}\right)$, the mathematical expression that characterizes conflict can be defined on $\mathbf{s}=\mathbf{s}_{o}-\mathbf{s}_{i}$ and $\mathbf{v}=\mathbf{v}_{o}-\mathbf{v}_{i}$, i.e., the relative position and velocity vectors, respectively, of the ownship with respect to the intruder. Therefore, conflict can be viewed as a Boolean condition on two vectors $\mathbf{s}$ and $\mathbf{v}$ rather than a condition on four vectors $\mathbf{s}_{o}, \mathbf{v}_{o}, \mathbf{s}_{i}$, and $\mathbf{v}_{i}$. This relative view simplifies the mathematical development presented in this paper. Thus, the Boolean condition conflict can be formally defined as follows.

$$
\operatorname{conflict}(\mathbf{s}, \mathbf{v}) \equiv \exists t \geq 0:\|\mathbf{s}+t \mathbf{v}\|<D .
$$

In this paper, the relative position and velocity vectors, $\mathbf{s}$ and $\mathbf{v}$, will commonly be used in place of $\mathbf{s}_{o}-\mathbf{s}_{i}$ and $\mathbf{v}_{o}-\mathbf{v}_{i}$, respectively.

As noted in Section II, a criterion for instantaneous maneuvers can be be analytically expressed by formulas involving the positions and velocity vectors of the two aircraft and a parameter that indicates directionality of the set of maneuvers, e.g., left/right, up/down, etc. An example of a criterion presented in [5] is horizontal_criterion. This criterion guarantees that any resolution maneuver will independently and coordinatedly solve a conflict between the aircraft. The criterion is defined as follows.

$$
\text { horizontal_criterion }(\mathbf{s}, \mathbf{v}, \epsilon)\left(\mathbf{v}^{\prime}\right) \equiv \mathbf{s} \cdot \mathbf{v}^{\prime} \geq \epsilon \frac{\sqrt{\mathbf{s} \cdot \mathbf{s}-D^{2}}}{D} \operatorname{det}\left(\mathbf{s}, \mathbf{v}^{\prime}\right) \text {. }
$$

Here, the direction parameter $\epsilon$ is either -1 or 1 . Figure 1 illustrates the set of velocity vectors that satisfy the criterion horizontal_criterion. The key property of this criterion is that it guarantees coordination when both aircraft use the same value of $\epsilon$. This value can be chosen implicitly without any communication between the aircraft. Given a choice of $\epsilon$, if each aircraft executes a maneuver that satisfies the criterion, then the combined result will both satisfy the criterion and remain conflict-free.

A criterion related to repulsiveness is relevant to the work presented in this paper. A resolution maneuver is repulsive if it increases the distance at the closest point of approach between two conflicting aircraft. Formally, this is defined as a condition on $\mathbf{s}, \mathbf{v}$, which are the current relative position and velocity of the aircraft, and $\mathbf{v}^{\prime}$, which is a relative velocity vector after a maneuver. Repuslive is formally is defined as follows.

$$
\begin{aligned}
\operatorname{repulsive}(\mathbf{s}, \mathbf{v})\left(\mathbf{v}^{\prime}\right) \equiv & \left(\mathbf{s} \cdot \mathbf{v}<0 \text { and }\left(\operatorname{tca}\left(\mathbf{s}, \mathbf{v}^{\prime}\right) \leq 0 \text { or }\|\mathbf{s}+\operatorname{tca}(\mathbf{s}, \mathbf{v}) \cdot \mathbf{v}\|<\left\|\mathbf{s}+\operatorname{tca}\left(\mathbf{s}, \mathbf{v}^{\prime}\right) \cdot \mathbf{v}^{\prime}\right\|\right)\right) \\
& \text { or }\left(\mathbf{s} \cdot \mathbf{v} \geq 0 \text { and }\left(\mathbf{v}=\mathbf{0} \Rightarrow \mathbf{s} \cdot \mathbf{v}^{\prime} \geq 0\right) \text { and }\left(\mathbf{v} \neq \mathbf{0} \Rightarrow \mathbf{s} \cdot \mathbf{v}^{\prime}>\mathbf{s} \cdot \mathbf{v}\right)\right) .
\end{aligned}
$$

This definition uses the function tca, which computes the time at closest point approach for converging aircraft with initial relative position $\mathbf{s}$ and relative velocity $\mathbf{v}$, and is defined as follows.

$$
\operatorname{tca}(\mathbf{s}, \mathbf{v}) \equiv-\frac{\mathbf{s} \cdot \mathbf{v}}{\|\mathbf{v}\|^{2}} .
$$


There are two main conditions in Formula (6), the first being for the case where the aircraft are originally converging (getting closer together), i.e., when $\mathbf{s} \cdot \mathbf{v}<0$, and the second being for the case where they are diverging, i.e. when $\mathbf{s} \cdot \mathbf{v} \geq 0$. Given the current relative position $\mathbf{s}=\mathbf{s}_{o}-\mathbf{s}_{i}$ and velocity $\mathbf{v}=\mathbf{v}_{o}-\mathbf{v}_{i}$, for the ownship aircraft with respect to the intruder, repulsive $(\mathbf{s}, \mathbf{v})$ is a condition on a relative velocity vector $\mathbf{v}^{\prime}=\mathbf{v}_{o}^{\prime}-\mathbf{v}_{i}$, where $\mathbf{v}_{o}^{\prime}$ is a new velocity vector representing a possible maneuver for the ownship. It implies that the minimum distance achieved by the aircraft for positive times increases when the new velocity vector $\mathbf{v}_{o}^{\prime}$ is chosen by the ownship instead of the current vector $\mathbf{v}_{o}$. The simplest visualization of repulsiveness is when two aircraft are in a head-on conflict scenario with a horizontal offset. In this case, repulsiveness just means that the new maneuver is incremental in the direction opposite the other aircraft, as indicated in Figure 2. A criterion that ensures repulsiveness is defined as follows.

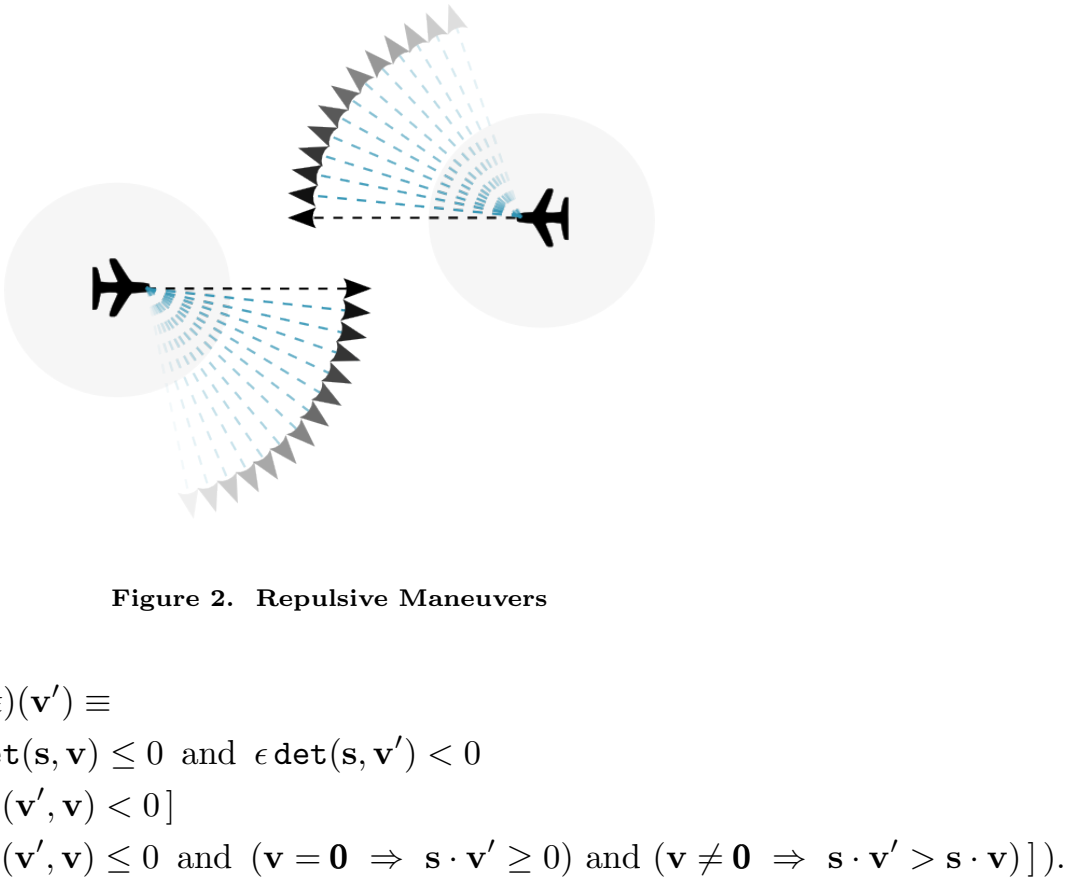

Just as in the definition of repulsion, repulsive_criterion has two main conditions, the first being for the case where the aircraft are originally converging, i.e., when $\mathbf{s} \cdot \mathbf{v}<0$, and the second being for the case where they are diverging, i.e. when $\mathbf{s} \cdot \mathbf{v} \geq 0$. Also, the $\epsilon$ parameter is similar to that used in the definition of horizontal_criterion above. It is equal to either -1 or 1 and indicates direction in the relative frame of reference. In general, the value for $\epsilon$ that should be used in practice is the sign of $\operatorname{det}(\mathbf{v}, \mathbf{s})$, which will be the same value when computed from the perspective of each aircraft. The following theorem, which states the coordination property of this criterion, has been formally proved in PVS.

Theorem 1. If both aircraft maneuver instantaneously and each maneuver satisfies repulsive_criterion, then the combined result is repulsive. That is, for all $\mathbf{s}_{o}, \mathbf{v}_{o}, \mathbf{v}_{o}^{\prime}, \mathbf{s}_{i}, \mathbf{v}_{i}, \mathbf{v}_{i}^{\prime}$ and $\epsilon$, if

- repulsive_criterion $\left(\mathbf{s}_{o}-\mathbf{s}_{i}, \mathbf{v}_{o}-\mathbf{v}_{i}, \epsilon\right)\left(\mathbf{v}_{o}^{\prime}-\mathbf{v}_{i}\right)$ and

- repulsive_criterion $\left(\mathbf{s}_{i}-\mathbf{s}_{o}, \mathbf{v}_{i}-\mathbf{v}_{o}, \epsilon\right)\left(\mathbf{v}_{i}^{\prime}-\mathbf{v}_{o}\right)$

both hold, then repulsive $\left(\mathbf{s}_{o}-\mathbf{s}_{i}, \mathbf{v}_{o}-\mathbf{v}_{i}\right)\left(\mathbf{v}_{o}^{\prime}-\mathbf{v}_{i}^{\prime}\right)$ holds as well.

The criterion repulsive_criterion is fundamental to the definition of the criterion for kinematic maneuvers presented in Section IV and the coordination theorem of repulsive_criterion implies the coordination of this more general kinematic criterion.

\section{Coordination for Kinematic Maneuvers}

If there is a conflict between the ownship and the intruder, or if the aircraft have already lost separation, a resolution maneuver will be taken by one or both aircraft to resolve the conflict or to regain adequate separation. Henceforth, these maneuvers are modeled by kinematic trajectories. 
A kinematic trajectory is represented as a sequence of velocities, along with a timestep value which specifies how long the aircraft have each velocity in the sequence. Formally, kinematic maneuvers are functions from the positive natural numbers $\mathbf{N}^{+}$into 2 -D velocity vectors. Let $\mathbf{V}: \mathbf{N}^{+} \rightarrow \mathbf{R}^{2}$ be one such function. It is assumed that there is an associated time step $\tau$ and that during the time interval $[(k-1) \cdot \tau, k \cdot \tau]$, where $k \in \mathbf{N}^{+}$, the aircraft executing the maneuver has velocity $\mathbf{V}(k)$. Moreover, there is assumed to be a number $N$ of time steps after which the aircraft follows its last velocity indefinitely. This piecewise linear trajectory can closely approximate a true trajectory, since every continuously differentiable function can be uniformly approximated on an interval by a piecewise linear function [8].

The ownship's and the intruder's kinematic maneuvers are denoted $\mathbf{V}_{o}$ and $\mathbf{V}_{i}$, respectively. It is assumed for mathematical simplicity that the time step value is the same for the ownship as for the intruder.

Example 2. If the ownship has initial position $\mathbf{s}_{o}=(0,0)$ and initial velocity $\mathbf{v}_{o}=(300,0)$ (the units do not matter) and makes a constant bank angle turn 90 degrees to the left by changing its heading by 1 degree per second, the corresponding maneuver $\mathbf{V}_{o}$ is given by

$$
\mathbf{V}_{o}(k)=\left(300 \cos \left(\frac{k \pi}{180}\right), 300 \sin \left(\frac{k \pi}{180}\right)\right),
$$

for $k \geq 1$, and the parameter $N$ is 90 . That is, after 90 incremental changes to its velocity, the ownship will continue indefinitely with velocity vector $(0,300)$.

Just as in the case of the current linear trajectories, where the relative velocity $\mathbf{v}$ can be used to compute the relative position at a future time $t$ as $\mathbf{s}+t \mathbf{v}$, the notion of a relative velocity sequence is useful when studying the coordination of kinematic maneuvers. The relative velocity sequences $\mathbf{V}_{o}-\mathbf{v}_{i}, \mathbf{v}_{o}-\mathbf{V}_{i}$, and $\mathbf{V}_{o}-\mathbf{V}_{i}$ are defined by

$$
\begin{aligned}
\left(\mathbf{V}_{o}-\mathbf{v}_{i}\right)(j) & \equiv \mathbf{V}_{o}(j)-\mathbf{v}_{i} \\
\left(\mathbf{v}_{o}-\mathbf{V}_{i}\right)(j) & \equiv \mathbf{v}_{o}-\mathbf{V}_{i}(j) \\
\left(\mathbf{V}_{o}-\mathbf{V}_{i}\right)(j) & \equiv \mathbf{V}_{o}(j)-\mathbf{V}_{i}(j) .
\end{aligned}
$$

The function pos_step, defined below, computes the sequence of positions so that pos_step $(\mathbf{s}, \mathbf{V}, \tau)(j)$ is the position at the moment velocity $\mathbf{V}(j)$ is assumed. Thus, pos_step $(\mathbf{s}, \tau, \mathbf{V})(1)$ is equal to the initial position s. Further, pos_step is defined recursively on $j$.

$$
\begin{aligned}
\operatorname{pos} \_s t e p(\mathbf{s}, \tau, \mathbf{V})(j) \equiv & \text { if } j=1 \text { then } \mathbf{s} \\
& \text { else pos_step }(\mathbf{s}, \tau, \mathbf{V})(j-1)+\tau \mathbf{V}(j-1) \\
& \text { endif. }
\end{aligned}
$$

By using the function pos_step, it is easy to compute the position at any future time $t$ by first determining which time interval $t$ falls into. This is accomplished through the function position, which computes the position at time $t$.

$$
\begin{aligned}
& \operatorname{position}(\mathbf{s}, \tau, \mathbf{V}, N)(t) \equiv \operatorname{let} j=\min \left(\operatorname{floor}\left(\frac{t}{\tau}\right)+1, N\right) \text { in } \\
& \text { pos_step }(\mathbf{s}, \tau, \mathbf{V})(j)+(t-\tau \cdot(j-1)) \mathbf{V}(j) \text {. }
\end{aligned}
$$

The concept of repulsive maneuvers is especially relevant for kinematic, piecewise linear maneuvers such as those represented by velocity sequences described above. As in the case of instantaneous maneuvers, a kinematic resolution maneuver is called repulsive if it increases the distance at the closest point of approach between the aircraft. It is defined as a condition on relative kinematic trajectories. Formally, a relative velocity sequence is repulsive if the distance between the aircraft at any future time is not less than the minimum distance between the aircraft along their current trajectories. If the aircraft are currently converging, then this minimum distance is attained at the time of closest approach tca $(\mathbf{s}, \mathbf{v})$, and if they are currently diverging, then this minimum is attained at time 0 . Repulsiveness for these kinematic maneuvers is formally defined as follows.

$$
\begin{aligned}
& \text { repulsive_kin(s, } \mathbf{v}, \tau, \mathbf{V}, N) \equiv \\
& \qquad \begin{array}{l}
(\mathbf{s} \cdot \mathbf{v}<0 \text { and } \forall t: t \geq 0 \Longrightarrow\|\operatorname{position}(\mathbf{s}, \tau, \mathbf{V}, N)(t)\| \geq\|\mathbf{s}+\operatorname{tca}(\mathbf{s}, \mathbf{v}) \mathbf{v}\|) \text { or } \\
(\mathbf{s} \cdot \mathbf{v} \geq 0 \text { and } \forall t: t \geq 0 \Longrightarrow\|\operatorname{position}(\mathbf{s}, \tau, \mathbf{V}, N)(t)\| \geq\|\mathbf{s}\|) .
\end{array}
\end{aligned}
$$


In contrast to Formula (6), Formula (12) can not be algorithmically evaluated due to the universal quantifier. As it turns out, just as the instantaneous criterion repulsive_criterion defined in Section III guaranteed coordinated repulsiveness, there is a criterion repulsive_kin_crit for these kinematic, piecewise linear relative velocity sequences that also guarantees coordinated repulsiveness when both aircraft maneuver. It is defined piecewise on each linear segment of the maneuver and, unlike the definition of repulsiveness given by Formula (12), it can be computed by an algorithm. The instantaneous criterion repulsive_criterion is used in the definition of this new criterion. The new criterion repulsive_kin_crit checks incrementally whether each velocity $\mathbf{V}(j+1)$ satisfies the criterion repulsive_criterion with respect to the previous velocity $\mathbf{V}(j)$, at the position given by pos_step $(\mathbf{s}, \tau, \mathbf{V})(j+1)$. It is formally defined as follows.

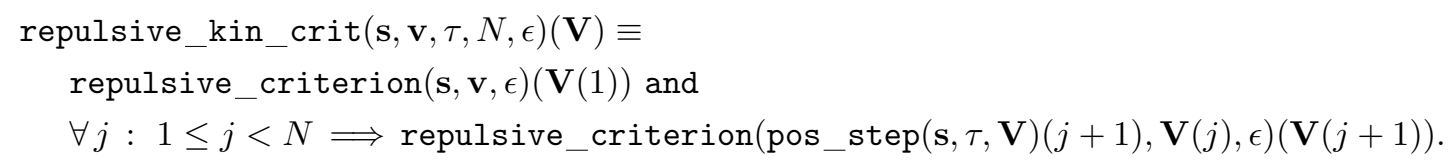

It can be seen that any relative velocity sequence $\mathbf{V}$ that satisfies this condition will also be repulsive. In fact, the following theorem has been formally proved in PVS.

Theorem 3 (Independence). repulsive_kin_crit(s, v, $\tau, N, \epsilon)(\mathbf{V}) \Longrightarrow$ repulsive_kin $(\mathbf{s}, \mathbf{v}, \tau, \mathbf{V}, N)$.

Example 4. Consider the scenario in Figure 3. In this case, the initial positions of the two aircraft are $\mathbf{s}_{o}=(10,2), \mathbf{s}_{i}=(0,0)$ (units are nautical miles), and the initial velocities are $\mathbf{v}_{o}=(-500,0), \mathbf{v}_{i}=(500,0)$ (units are knots). In this scenario, the ownship executes an avoidance maneuver by changing its heading by

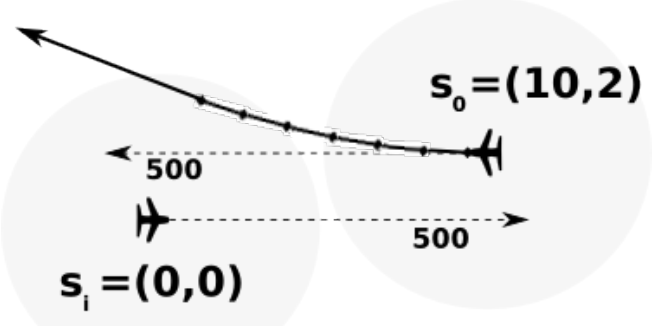

Figure 3. Repulsive Relative Velocity Sequence

3 degrees every 10 seconds for 1 minute and then continuing linearly. This sequence of velocity changes is represented by

$$
\mathbf{V}_{o}(j)=\left(-500 \cos \left(\frac{j \pi}{60}\right), 500 \sin \left(\frac{j \pi}{60}\right)\right)
$$

the corresponding time step $\tau$ is 10 seconds, or $\tau=0.002 \overline{7}$ hours, and finally the corresponding value for $N$ is 7 . It can be seen that for these values, repulsive_kin_crit $\left(\mathbf{s}_{o}-\mathbf{s}_{i}, \mathbf{v}_{o}-\mathbf{v}_{i}, \tau, N, \epsilon\right)\left(\mathbf{V}_{o}-\mathbf{v}_{i}\right)$ holds for the relative velocity sequence $\mathbf{V}_{o}-\mathbf{v}_{i}$ when $\epsilon=1$, which represents the right direction from the point of view of each aircraft, but does not hold when $\epsilon=-1$, which represents the left direction. Also, it is clear from the definition of repulsiveness that repulsive_kin $\left(\mathbf{s}_{o}-\mathbf{s}_{i}, \mathbf{v}_{o}-\mathbf{v}_{i}, \tau, \mathbf{V}_{o}-\mathbf{v}_{i}, N\right)$ holds for this relative velocity sequence as well.

The most important property of the criterion repulsive_kin_crit is that, in addition to guaranteeing repulsiveness when only one aircraft maneuvers, it also guarantees repulsiveness when both aircraft maneuver, which means that it is coordinated with respect to repulsiveness. The following theorem, which states the main coordination property for this criterion, has been formally proved in PVS.

Theorem 5 (Coordination). If the conditions

- repulsive_kin_crit $\left(\mathbf{s}_{o}-\mathbf{s}_{i}, \mathbf{v}_{o}-\mathbf{v}_{i}, \tau, N, \epsilon\right)\left(\mathbf{V}_{o}-\mathbf{v}_{i}\right)$
- repulsive_kin_crit $\left(\mathbf{s}_{i}-\mathbf{s}_{o}, \mathbf{v}_{i}-\mathbf{v}_{o}, \tau, N, \epsilon\right)\left(\mathbf{V}_{i}-\mathbf{v}_{o}\right)$ 
both hold, then relative velocity sequence $\mathbf{V}_{o}-\mathbf{V}_{i}$ is repulsive. That is, the condition

$$
\text { repulsive_kin }\left(\mathbf{s}_{o}-\mathbf{s}_{i}, \mathbf{v}_{o}-\mathbf{v}_{i}, \tau, \mathbf{V}_{o}-\mathbf{V}_{i}, N\right)
$$

holds.

Example 6. Consider the same scenario that was given in Figure 3, but in the case where both aircraft execute similar maneuvers. As above, the initial positions of the two aircraft are $\mathbf{s}_{o}=(10,2), \mathbf{s}_{i}=(0,0)$, and the initial velocities are $\mathbf{v}_{o}=(-500,0), \mathbf{v}_{i}=(500,0)$, and the time step is $\tau=0.002 \overline{7}$ hours (10 seconds). In this scenario, each aircraft executes an avoidance maneuver by changing its heading to the right

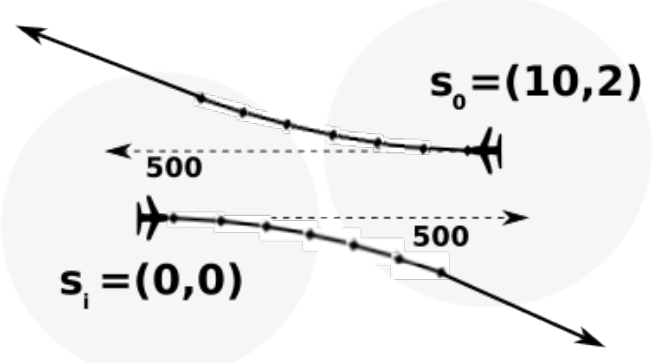

Figure 4. Repulsive Coordinated Relative Velocity Sequence

by 3 degrees every 10 seconds for 1 minute and then continuing linearly. This sequences of velocity changes are represented by

$$
\mathbf{V}_{o}(j)=\left(-500 \cos \left(\frac{j \pi}{60}\right), 500 \sin \left(\frac{j \pi}{60}\right)\right) \quad \text { and } \quad \mathbf{V}_{o}(j)=\left(500 \cos \left(\frac{j \pi}{60}\right),-500 \sin \left(\frac{j \pi}{60}\right)\right) .
$$

For these values, repulsive_kin_crit $\left(\mathbf{s}_{o}-\mathbf{s}_{i}, \mathbf{v}_{o}-\mathbf{v}_{i}, \tau, N, \epsilon\right)\left(\mathbf{V}_{o}-\mathbf{v}_{i}\right)$ and repulsive_kin_crit $\left(\mathbf{s}_{i}-\right.$ $\left.\mathbf{s}_{o}, \mathbf{v}_{i}-\mathbf{v}_{o}, \tau, N, \epsilon\right)\left(\mathbf{V}_{i}-\mathbf{v}_{o}\right)$ both hold when $\epsilon=1$, which represents the right direction from the point of view of each aircraft, but do not hold when $\epsilon=-1$, which represents the left direction. Also, it is clear from the definition of repulsiveness that repulsive_kin $\left(\mathbf{s}_{o}-\mathbf{s}_{i}, \mathbf{v}_{o}-\mathbf{v}_{i}, \tau, \mathbf{V}_{o}-\mathbf{V}_{i}, N\right)$ holds for this relative velocity sequence as well, and thus these two maneuvers exhibit coordination.

Theorem 5 requires that both aircraft choose the same value for $\epsilon$. However, this is quite easy even without explicit communication. In fact, $\epsilon$ can be simply chosen by the ownship to be the sign of $\operatorname{det}\left(\mathbf{v}_{o}-\mathbf{v}_{i}, \mathbf{s}_{o}-\mathbf{s}_{i}\right)$. The intruder will similarly choose $\epsilon$ to be the sign of $\operatorname{det}\left(\mathbf{v}_{i}-\mathbf{v}_{o}, \mathbf{s}_{i}-\mathbf{s}_{o}\right)$, which is exactly the same value since

$$
\operatorname{det}\left(\mathbf{v}_{o}-\mathbf{v}_{i}, \mathbf{s}_{o}-\mathbf{s}_{i}\right)=\operatorname{det}\left(\mathbf{v}_{i}-\mathbf{v}_{o}, \mathbf{s}_{i}-\mathbf{s}_{o}\right) .
$$

In general, except in the case where the aircraft are on perfect straight line collision trajectories, this choice of $\epsilon$ is the only choice possible while still satisfying the criterion repulsive_kin_crit. Indeed, the condition inside the definition of repulsive_kin_crit requires, by the definition of repulsive_criterion, that $\epsilon \operatorname{det}\left(\mathbf{s}_{o}-\mathbf{s}_{i}, \mathbf{v}_{o}-\mathbf{v}_{i}\right) \leq 0$. Unless $\operatorname{det}\left(\mathbf{s}_{o}-\mathbf{s}_{i}, \mathbf{v}_{o}-\mathbf{v}_{i}\right)=0$, which is precisely when the aircraft will eventually collide, this is only possible when $\epsilon$ is the sign of $\operatorname{det}\left(\mathbf{v}_{o}-\mathbf{v}_{i}, \mathbf{s}_{o}-\mathbf{s}_{i}\right)$.

Another important note regarding Theorem 5 is that Figure 4 is just a simple example of coordination where it is clear what each aircraft should do. More complicated encounter geometries exist where it is unclear at first glance which direction each aircraft should maneuver to incrementally improve the situation. Theorem 5 guarantees that even in these complicated scenarios, if each aircraft chooses a maneuver that satisfies the criterion repulsive_kin_crit, the combined result will still exhibit coordination when both aircraft maneuver. The theorem holds in all possible geometries.

Theorem 5 guarantees implicit coordination in the sense that the aircraft do not need to communicate anything other than their original positions and velocities for the kinematic maneuvers to exhibit coordination. Implicit coordination is sometimes viewed as unnecessary when communication channels already exist in a concept of operations. However, it should be noted that implicit coordination such as that guaranteed by this theorem can provide an extra layer of safety even when such communication is possible. This is because it can guarantee coordination even when communication fails or messages are dropped, as long as the aircraft have accurate estimates of each other's current positions and velocities. 


\section{An Interesting Example}

Recall that the kinematic criterion defined in Section IV is defined by requiring each successive velocity in the relative velocity sequence $\mathbf{V}$ to satisfy the instantaneous repulsive criterion repulsive_criterion. It may seem obvious that requiring each incremental change in the velocity sequence to satisfy a safety property like repulsiveness at each incremental step is enough to ensure coordination even when both aircraft maneuver. However, this is not always the case. An example is given below in which the aircraft are originally diverging, and the maneuver that each aircraft executes is incrementally diverging from the other aircraft's current linear trajectory, but if they both maneuver, the combination of the maneuvers puts the aircraft in conflict. This is similar to a phenomenon with instantaneous maneuvers that, in the case where the aircraft are originally diverging, allows each aircraft to turn toward the other aircraft, thus losing coordination but still maintaining safety in the case where only one of them maneuvers. That example for instantaneous maneuvers was presented in [5].

The example presented below shows that it is not enough to design the instantaneous repulsive criterion repulsive_criterion, defined in Section III, to simply ensure divergence in the case where the aircraft are initially diverging. The authors initially defined the kinematic criterion repulsive_kin_crit using that condition, namely that in the case where the states were diverging, each successive velocity in the maneuver was only required to be divergent. The process of trying to formally prove the coordination properties of this kinematic criterion failed, leading the authors to realize that this definition of the criterion was too liberal in the diverging case. A more conservative version of the instantaneous repulsive criterion repulsive_criterion was needed to ensure the coordination of the kinematic criterion.

In this example, the aircraft have the following initial states: $\mathbf{s}_{o}=(-10,0), \mathbf{v}_{o}=(-300,0), \mathbf{s}_{i}=(10,0)$, and $\mathbf{v}_{i}=(300,0)$. The units for positions are nautical miles $(\mathrm{nm})$ and the units for velocities are knots. The two aircraft are initially separated by $20 \mathrm{~nm}$ and are heading in exactly opposite directions. Thus, they are diverging. The initial states are shown in Figure 5. In this figure, the ownship is shown in blue and the intruder in green.

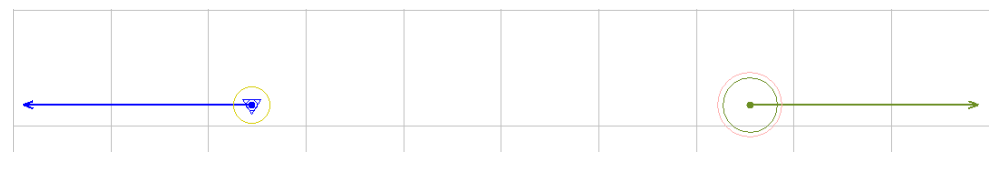

Figure 5. Initial States Diverging

Each aircraft in this example executes a turn with a constant bank angle of 25 degrees, the ownship to its right and the intruder to its left. This results in turns with heading changes of approximately 1.7 degrees per second. Each aircraft executes the turn until its track angle has changed 165 degrees from its initial track angle, at which point it continues linearly. These maneuvers can be seen in Figure 6. Each incremental

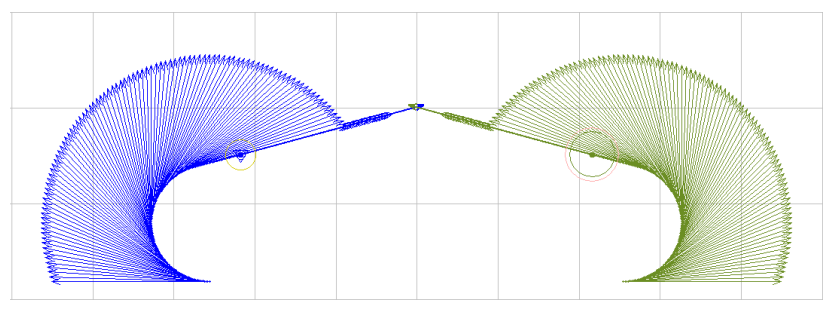

Figure 6. Divergent But Not Coordinated Maneuvers

velocity change by the ownship along its maneuver maintains divergence from the intruder, assuming that the intruder continues its originally linear trajectory without turning. The same holds for the intruder if the ownship continues its original trajectory. However, if they both maneuvered at the same time, the aircraft would collide at approximately 230.5 seconds after the initial states. This shows that for the safety that coordination ensures, the definition of the instantaneous repulsive criterion must restrict maneuvers in the initially-divergent case more than just requiring continued divergence. This is the reason for the more 
elaborate conditions in Formula 6 in Section III in the divergence case, i.e., when $\mathbf{s} \cdot \mathbf{v} \geq 0$, which require

$$
\epsilon \operatorname{det}\left(\mathbf{v}^{\prime}, \mathbf{v}\right) \leq 0 \text { and }\left(\mathbf{v}=\mathbf{0} \Rightarrow \mathbf{s} \cdot \mathbf{v}^{\prime} \geq 0\right) \text { and }\left(\mathbf{v} \neq \mathbf{0} \Rightarrow \mathbf{s} \cdot \mathbf{v}^{\prime}>\mathbf{s} \cdot \mathbf{v}\right)
$$

\section{Conclusion}

This paper extends previous work $[4,5]$ on coordination for instantaneous maneuvers. That work focused mostly on conflict resolution, where distances between the aircraft often make the instantaneous maneuver assumption a reasonable approximation. However, for cases where the time to conflict is small, or alternatively for recovery from loss of separation, the piecewise linear kinematic model used in this paper provides a better approximation of actual aircraft trajectories. This model is also better at determining whether coordinated behavior will actually be observed by aircraft executing those maneuvers.

This paper presents the notion of a criterion for kinematic, piecewise linear maneuvers that guarantees independence and implicit coordination with respect to repulsiveness. Implicit coordination can be useful for ensuring safe interplay of maneuvers between aircraft, because aircraft do not need to communicate anything other than their original positions and velocities for the kinematic maneuvers to exhibit coordination. It is also important to note that implicit coordination can enhance safety even when aircraft communicate more information about their intent. In that case, it can add an extra layer of safety, because it can guarantee coordination even when communication fails or messages are dropped, as long as the aircraft have accurate estimates of each other's current positions and velocities.

The primary motivation behind the further development of the concept of implicit coordination is that it enhances safety features key to any separation assurance system. Thus, the theorems presented here have been proved correct in an interactive theorem prover, which is a system that allows safety properties to be stated and formally proved. The use of an interactive theorem prover is justified by the paramount importance of safety in new air traffic management systems.

\section{References}

\footnotetext{
${ }^{1}$ Ricky Butler and César Muñoz. Formally verified practical algorithms for recovery from loss of separation. Technical Memorandum NASA/TM-2009-215726, NASA, Langley Research Center, Hampton VA 23681-2199, USA, June 2009.

${ }^{2}$ United States Government. General operating and flight rules, 14CFR91.113, 2012.

${ }^{3}$ James Kuchar and Lee Yang. A review of conflict detection and resolution modeling methods. IEEE Transactions on Intelligent Transportation Systems, 1(4):179-189, December 2000.

${ }^{4}$ César Muñoz, Ricky Butler, Anthony Narkawicz, Jeffrey Maddalon, and George Hagen. A criteria standard for conflict resolution: A vision for guaranteeing the safety of self-separation in NextGen. Technical Memorandum NASA/TM-2010-216862, NASA, Langley Research Center, Hampton VA 23681-2199, USA, October 2010.

${ }^{5}$ Anthony Narkawicz and César Muñoz. State-based implicit coordination and applications. Technical Publication NASA/TP-2011-217067, NASA, Langley Research Center, Hampton VA 23681-2199, USA, March 2011.

${ }^{6}$ Sam Owre, John Rushby, and Natarajan Shankar. PVS: A prototype verification system. In Deepak Kapur, editor, Proceeding of the 11th International Conference on Automated Deductioncade, volume 607 of Lecture Notes in Artificial Intelligence, pages 748-752. Springer, June 1992.

${ }^{7}$ RTCA SC-147. RTCA-DO-185B, Minimum operational performance standards for traffic alert and collision avoidance system II (TCAS II), July 2009.

${ }^{8}$ Walter Rudin. Principles of Mathematical Analysis. McGraw-Hill, third edition, 1976.

${ }^{9}$ David J. Wing and William B Cotton. Autonomous flight rules: A concept for self-separation in u.s domestic airspace. Technical Memorandum NASA/TP-2011-217174, NASA, Langley Research Center, Hampton VA 23681-2199, USA, November 2011.
} 